\title{
Proton Electromagnetic Form Factors Data Are in Disagreement with New Electron-Positron Annihilation into Proton-Antiproton Pair Total Cross Section Measurements
}

\author{
Anna Zuzana Dubnickova ${ }^{1}$, Stanislav Dubnicka ${ }^{2, *}$ \\ ${ }^{1}$ Department of Theoretical Physics, Comenius University, Bratislava, Slovak Republic \\ ${ }^{2}$ Institute of Physics, Slovak Academy of Sciences, Bratislava, Slovak Republic
}

\section{Email address:}

dubnickova@fmph.uniba.sk (A. Z. Dubnickova), stanislav.dubnicka@savba.sk (S. Dubnicka)

${ }^{*}$ Corresponding author

\section{To cite this article:}

Anna Zuzana Dubnickova, Stanislav Dubnicka. Proton Electromagnetic Form Factors Data Are in Disagreement with New Electron-Positron Annihilation into Proton-Antiproton Pair Total Cross Section Measurements. American Journal of Modern Physics.

Vol. 10, No. 3, 2021, pp. 41-50. doi: 10.11648/j.ajmp.20211003.11

Received: March 30, 2021; Accepted: April 16, 2021; Published: May 7, 2021

\begin{abstract}
In this paper a consistency of new very precise data on electron-positron annihilation into proton-antiproton pair total cross section with existing proton and neutron electromagnetic form factors data is investigated. First, the result is represented by a theoretically predicted dashed line for the total cross section obtained in the simultaneous analysis of the proton and neutron form factors data by the advanced nucleon electromagnetic structure Unitary and Analytic model, and then, as the neutron data are always less precise from objective reasons than the proton data, the result is also represented by a theoretically predicted full line for the total cross section obtained in the analysis of only the proton form factors data in space-like and time-like regions by the advanced proton electromagnetic structure Unitary and Analytic model. In both cases one finds disagreement between the electron-positron annihilation into proton-antiproton pair total cross section data and the corresponding form factors data, which is demonstrated by a disagreement of the dashed and full curves representing theoretically predicted electron-positron annihilation into proton-antiproton pair total cross sections behaviors.
\end{abstract}

Keywords: Nucleons, Vector Mesons, Electromagnetic Form Factors, Analyticity, Cross Sections

\section{Introduction}

The electromagnetic (EM) structure of the nucleon (isodoublet compound of the proton and neutron) is completely described theoretically by two independent functions of one variable, the Dirac $F_{1}^{N}(t)$ and Pauli $F_{2}^{N}(t)$ form factors (FFs), which naturally appear in a decomposition of the nucleon matrix element of the EM current $J_{\mu}^{E M}(0)$ as coefficients of two linearly independent covariants constructed from the four momenta $p, p^{\prime}, \gamma$-matrices and Dirac bi-spinors

$$
\begin{aligned}
& <N\left|J_{\mu}^{E M}(0)\right| N>= \\
& \quad e \bar{u}\left(p^{\prime}\right)\left[\gamma_{\mu} F_{1}^{N}(t)+\frac{i}{2 m_{N}} \sigma_{\mu \nu}\left(p^{\prime}-p\right)_{\mu} F_{2}^{N}(t)\right] u(p),
\end{aligned}
$$

with $m_{N}$ to be the nucleon mass.

A description of EM structure of the nucleon is even improved if mixed transformation properties of the EM current $J_{\mu}^{E M}(0)$ under the rotation in the isospin space is utilized. A part of $J_{\mu}^{E M}(0)$ transforms as an isoscalar and its another part as the third component of isovector. The latter leads to a splitting of the proton and neutron Dirac and Pauli EM FFs to flavour-independent isoscalar and isovector parts $F_{1 s}^{N}(t), F_{1 v}^{N}(t), F_{2 s}^{N}(t), F_{2 v}^{N}(t)$ as follows

$$
\begin{aligned}
& F_{1}^{p}(t)=\left[F_{1 s}^{N}(t)+F_{1 v}^{N}(t)\right], \\
& F_{2}^{p}(t)=\left[F_{2 s}^{N}(t)+F_{2 v}^{N}(t)\right], \\
& F_{1}^{n}(t)=\left[F_{1 s}^{N}(t)-F_{1 v}^{N}(t)\right], \\
& F_{2}^{n}(t)=\left[F_{2 s}^{N}(t)-F_{2 v}^{N}(t)\right],
\end{aligned}
$$

whereby the sign between them is specified by the sign of the third component of the isospin of the concrete nucleon under 
consideration.

The FFs $F_{1 s}^{N}(t), F_{1 v}^{N}(t), F_{2 s}^{N}(t), F_{2 v}^{N}(t)$ are analytic in the whole complex t-plane besides cuts on the positive real axis starting for isovector FFs at the two-pion threshold and for isoscalar FFs at three-pion threshold. In the paper [1] the advanced 9 vector-meson resonance Unitary and Analytic $(U \& A)$ model for nucleon isoscalar and isovector Dirac and Pauli FFs has been constructed

$$
\begin{aligned}
& F_{1 s}^{N}[V(t)]=\left(\frac{1-V^{2}}{1-V_{N}^{2}}\right)^{4}\left\{\frac{1}{2} H_{\omega^{\prime \prime}}(V) H_{\phi^{\prime \prime}}(V)\right. \\
& +\left[H_{\phi^{\prime \prime}}(V) H_{\omega^{\prime}}(V) \frac{\left(C_{\phi^{\prime \prime}}^{1 s}-C_{\omega^{\prime}}^{1 s}\right)}{\left(C_{\phi^{\prime \prime}}^{1 s}-C_{\omega^{\prime \prime}}^{1 s}\right)}+H_{\omega^{\prime \prime}}(V) H_{\omega^{\prime}}(V) \frac{\left(C_{\omega^{\prime \prime}}^{1 s}-C_{\omega^{\prime}}^{1 s}\right)}{\left(C_{\omega^{\prime \prime}}^{1 s}-C_{\phi^{\prime \prime}}^{1 s}\right)}-H_{\omega^{\prime \prime}}(V) H_{\phi^{\prime \prime}}(V)\right]\left(f_{\omega^{\prime} N N}^{(1)} / f_{\omega^{\prime}}\right) \\
& +\left[H_{\phi^{\prime \prime}}(V) H_{\phi^{\prime}}(V) \frac{\left(C_{\phi^{\prime \prime}}^{1 s}-C_{\phi^{\prime}}^{1 s}\right)}{\left(C_{\phi^{\prime \prime}}^{1 s}-C_{\omega^{\prime \prime}}^{1 s}\right)}+H_{\omega^{\prime \prime}}(V) H_{\phi^{\prime}}(V) \frac{\left(C_{\omega^{\prime \prime}}^{1 s}-C_{\phi^{\prime}}^{1 s}\right)}{\left(C_{\omega^{\prime \prime}}^{1 s}-C_{\phi^{\prime \prime}}^{1 s}\right)}-H_{\omega^{\prime \prime}}(V) H_{\phi^{\prime \prime}}(V)\right]\left(f_{\phi^{\prime} N N}^{(1)} / f_{\phi^{\prime}}\right) \\
& +\left[H_{\phi^{\prime \prime}}(V) L_{\omega}(V) \frac{\left(C_{\phi^{\prime \prime}}^{1 s}-C_{\omega}^{1 s}\right)}{\left(C_{\phi^{\prime \prime}}^{1 s}-C_{\omega^{\prime \prime}}^{1 s}\right)}+H_{\omega^{\prime \prime}}(V) L_{\omega}(V) \frac{\left(C_{\omega^{\prime \prime}}^{1 s}-C_{\omega}^{1 s}\right)}{\left(C_{\omega^{\prime \prime}}^{1 s}-C_{\phi^{\prime \prime}}^{1 s}\right)}-H_{\omega^{\prime \prime}}(V) H_{\phi^{\prime \prime}}(V)\right]\left(f_{\omega N N}^{(1)} / f_{\omega}\right) \\
& \left.+\left[H_{\phi^{\prime \prime}}(V) L_{\phi}(V) \frac{\left(C_{\phi^{\prime \prime}}^{1 s}-C_{\phi}^{1 s}\right)}{\left(C_{\phi^{\prime \prime}}^{1 s}-C_{\omega^{\prime \prime}}^{1 s}\right)}+H_{\omega^{\prime \prime}}(V) L_{\phi}(V) \frac{\left(C_{\omega^{\prime \prime}}^{1 s}-C_{\phi}^{1 s}\right)}{\left(C_{\omega^{\prime \prime}}^{1 s}-C_{\phi^{\prime \prime}}^{1 s}\right)}-H_{\omega^{\prime \prime}}(V) H_{\phi^{\prime \prime}}(V)\right]\left(f_{\phi N N}^{(1)} / f_{\phi}\right)\right\}
\end{aligned}
$$

with 5 free parameters $\left(f_{\omega^{\prime} N N}^{(1)} / f_{\omega^{\prime}}\right),\left(f_{\phi^{\prime} N N}^{(1)} / f_{\phi^{\prime}}\right),\left(f_{\omega N N}^{(1)} / f_{\omega}\right),\left(f_{\phi N N}^{(1)} / f_{\phi}\right), t_{i n}^{1 s}$,

$$
\begin{aligned}
F_{1 v}^{N}[W(t)] & =\left(\frac{1-W^{2}}{1-W_{N}^{2}}\right)^{4}\left\{\frac{1}{2} L_{\rho}(W) L_{\rho^{\prime}}(W)+\left[L_{\rho^{\prime}}(W) L_{\rho^{\prime \prime}}(W) \frac{\left(C_{\rho^{\prime}}^{1 v}-C_{\rho^{\prime \prime}}^{1 v}\right)}{\left(C_{\rho^{\prime}}^{1 v}-C_{\rho}^{1 v}\right)}\right.\right. \\
& \left.\left.+L_{\rho}(W) L_{\rho^{\prime \prime}}(W) \frac{\left(C_{\rho}^{1 v}-C_{\rho^{\prime \prime}}^{1 v}\right)}{\left(C_{\rho}^{1 v}-C_{\rho^{\prime}}^{1 v}\right)}-L_{\rho}(W) L_{\rho^{\prime}}(W)\right]\left(f_{\rho N N}^{(1)} / f_{\rho}\right)\right\},
\end{aligned}
$$

with two free parameters $\left(f_{\rho N N}^{(1)} / f_{\rho}\right)$ and $t_{i n}^{1 v}$,

$$
\begin{aligned}
& F_{2 s}^{N}[U(t)]=\left(\frac{1-U^{2}}{1-U_{N}^{2}}\right)^{6}\left\{\frac{1}{2}\left(\mu_{p}+\mu_{n}-1\right) H_{\omega^{\prime \prime}}(U) H_{\phi^{\prime \prime}}(U) H_{\omega^{\prime}}(U)\right. \\
& +\left[H_{\phi^{\prime \prime}}(U) H_{\omega^{\prime}}(U) H_{\phi^{\prime}}(U) \frac{\left(C_{\phi^{\prime \prime}}^{2 s}-C_{\phi^{\prime}}^{2 s}\right)\left(C_{\omega^{\prime}}^{2 s}-C_{\phi^{\prime}}^{2 s}\right)}{\left(C_{\phi^{\prime \prime}}^{2 s}-C_{\omega^{\prime \prime}}^{2 s}\right)\left(C_{\omega^{\prime}}^{2 s}-C_{\omega^{\prime \prime}}^{2 s}\right)}+H_{\omega^{\prime \prime}}(U) H_{\omega^{\prime}}(U) H_{\phi^{\prime}}(U) \frac{\left(C_{\omega^{\prime \prime}}^{2 s}-C_{\phi^{\prime}}^{2 s}\right)\left(C_{\omega^{\prime}}^{2 s}-C_{\phi^{\prime}}^{2 s}\right)}{\left(C_{\omega^{\prime \prime}}^{2 s}-C_{\phi^{\prime \prime}}^{2 s}\right)\left(C_{\omega^{\prime}}^{2 s}-C_{\phi^{\prime \prime}}^{2 s}\right)}\right. \\
& \left.+H_{\omega^{\prime \prime}}(U) H_{\phi^{\prime \prime}}(U) H_{\phi^{\prime}}(U) \frac{\left(C_{\omega^{\prime \prime}}^{2 s}-C_{\phi^{\prime}}^{2 s}\right)\left(C_{\phi^{\prime \prime}}^{2 s}-C_{\phi^{\prime}}^{2 s}\right)}{\left(C_{\omega^{\prime \prime}}^{2 s}-C_{\omega^{\prime}}^{2 s}\right)\left(C_{\phi^{\prime \prime}}^{2 s}-C_{\omega^{\prime}}^{2 s}\right)}-H_{\omega^{\prime \prime}}(U) H_{\phi^{\prime \prime}}(U) H_{\omega^{\prime}}(U)\right]\left(f_{\phi^{\prime} N N}^{(2)} / f_{\phi^{\prime}}\right) \\
& +\left[H_{\phi^{\prime \prime}}(U) H_{\omega^{\prime}}(U) L_{\omega}(U) \frac{\left(C_{\phi^{\prime \prime}}^{2 s}-C_{\omega}^{2 s}\right)\left(C_{\omega^{\prime}}^{2 s}-C_{\omega}^{2 s}\right)}{\left(C_{\phi^{\prime \prime}}^{2 s}-C_{\omega^{\prime \prime}}^{2 s}\right)\left(C_{\omega^{\prime}}^{2 s}-C_{\omega^{\prime \prime}}^{2 s}\right)}+H_{\omega^{\prime \prime}}(U) H_{\omega^{\prime}}(U) L_{\omega}(U) \frac{\left(C_{\omega^{\prime \prime}}^{2 s}-C_{\omega}^{2 s}\right)\left(C_{\omega^{\prime}}^{2 s}-C_{\omega}^{2 s}\right)}{\left(C_{\omega^{\prime \prime}}^{2 s}-C_{\phi^{\prime \prime}}^{2 s}\right)\left(C_{\omega^{\prime}}^{2 s}-C_{\phi^{\prime \prime}}^{2 s}\right)}\right. \\
& \left.+H_{\omega^{\prime \prime}}(U) H_{\phi^{\prime \prime}}(U) L_{\omega}(U) \frac{\left(C_{\omega^{\prime \prime}}^{2 s}-C_{\omega}^{2 s}\right)\left(C_{\phi^{\prime}}^{2 s}-C_{\omega}^{2 s}\right)}{\left(C_{\omega^{\prime \prime}}^{2 s}-C_{\omega^{\prime}}^{2 s}\right)\left(C_{\phi^{\prime \prime}}^{2 s}-C_{\omega^{\prime}}^{2 s}\right)}-H_{\omega^{\prime \prime}}(U) H_{\phi^{\prime \prime}}(U) H_{\omega^{\prime}}(U)\right]\left(f_{\omega N N}^{(2)} / f_{\omega}\right) \\
& +\left[H_{\phi^{\prime \prime}}(U) H_{\omega^{\prime}}(U) L_{\phi}(U) \frac{\left(C_{\phi^{\prime \prime}}^{2 s}-C_{\phi}^{2 s}\right)\left(C_{\omega^{\prime}}^{2 s}-C_{\phi}^{2 s}\right)}{\left(C_{\phi^{\prime \prime}}^{2 s}-C_{\omega^{\prime \prime}}^{2 s}\right)\left(C_{\omega^{\prime}}^{2 s}-C_{\omega^{\prime \prime}}^{2 s}\right)}+H_{\omega^{\prime \prime}}(U) H_{\omega^{\prime}}(U) L_{\phi}(U) \frac{\left(C_{\omega^{\prime \prime}}^{2 s}-C_{\phi}^{2 s}\right)\left(C_{\omega^{\prime}}^{2 s}-C_{\phi}^{2 s}\right)}{\left(C_{\omega^{\prime \prime}}^{2 s}-C_{\phi^{\prime \prime}}^{2 s}\right)\left(C_{\omega^{\prime}}^{2 s}-C_{\phi^{\prime \prime}}^{2 s}\right)}\right. \\
& \left.\left.+H_{\omega^{\prime \prime}}(U) H_{\phi^{\prime \prime}}(U) L_{\phi}(U) \frac{\left(C_{\omega^{\prime \prime}}^{2 s}-C_{\phi}^{2 s}\right)\left(C_{\phi^{\prime \prime}}^{2 s}-C_{\phi}^{2 s}\right)}{\left(C_{\omega^{\prime \prime}}^{2 s}-C_{\omega^{\prime}}^{2 s}\right)\left(C_{\phi^{\prime \prime}}^{2 s}-C_{\omega^{\prime}}^{2 s}\right)}-H_{\omega^{\prime \prime}}(U) H_{\phi^{\prime \prime}}(U) H_{\omega^{\prime}}(U)\right]\left(f_{\phi N N}^{2 s} / f_{\phi}\right)\right\}
\end{aligned}
$$

with four free parameters $\left(f_{\phi^{\prime} N N}^{(2)} / f_{\phi^{\prime}}\right),\left(f_{\omega N N}^{(2)} / f_{\omega}\right),\left(f_{\phi N N}^{(2)} / f_{\phi}\right), t_{i n}^{2 s}$, and

$$
F_{2 v}^{N}[X(t)]=\left(\frac{1-X^{2}}{1-X_{N}^{2}}\right)^{6}\left\{\frac{1}{2}\left(\mu_{p}-\mu_{n}-1\right) L_{\rho}(X) L_{\rho^{\prime}}(X) H_{\rho^{\prime \prime}}(X)\right\},
$$


dependent on only one free parameter $t_{i n}^{2 v}$, where

$$
V(t)=i \frac{\sqrt{\left(\frac{t_{i n}^{1 s}-t_{0}^{1 s}}{t_{0}^{1 s}}\right)^{1 / 2}+\left(\frac{t-t_{0}^{1 s}}{t_{0}^{1 s}}\right)^{1 / 2}}-\sqrt{\left(\frac{t_{i n}^{1 s}-t_{0}^{1 s}}{t_{0}^{1 s}}\right)^{1 / 2}-\left(\frac{t-t_{0}^{1 s}}{t_{0}^{1 s}}\right)^{1 / 2}}}{\sqrt{\left(\frac{t_{i n}^{1 s}-t_{0}^{1 s}}{t_{0}^{1 s}}\right)^{1 / 2}+\left(\frac{t-t_{0}^{1 s}}{t_{0}^{1 s}}\right)^{1 / 2}}+\sqrt{\left(\frac{t_{i n}^{1 s}-t_{0}^{1 s}}{t_{0}^{1 s}}\right)^{1 / 2}-\left(\frac{t-t_{0}^{1 s}}{t_{0}^{1 s}}\right)^{1 / 2}}},
$$

similarly $W(t), U(t), X(t)$, are conformal mappings of the corresponding four-sheeted Riemann surfaces in $t$ variable always into one $V-, W-, U-, X-$ plane. The $t_{0}^{1 s}=9 \mathrm{~m}_{\pi}^{2}$, $t_{0}^{1 v}=4 m_{\pi}^{2}, t_{0}^{2 s}=9 m_{\pi}^{2}, t_{0}^{2 v}=4 m_{\pi}^{2}$ are the lowest branch points and $t_{i n}^{1 s}, t_{i n}^{1 v}, t_{i n}^{2 s}, t_{i n}^{2 v}$ are the effective inelastic square root branch points, representing contributions of all possible higher inelastic thresholds effectively and therefore they are left in the analysis of data as free parameters.

Denotations $L$ (lower) and $H$ (higher)

$$
\begin{aligned}
& L_{r}(V)=\frac{\left(V_{N}-V_{r}\right)\left(V_{N}-V_{r}^{*}\right)\left(V_{N}-1 / V_{r}\right)\left(V_{N}-1 / V_{r}^{*}\right)}{\left(V-V_{r}\right)\left(V-V_{r}^{*}\right)\left(V-1 / V_{r}\right)\left(V-1 / V_{r}^{*}\right)}, \\
& C_{r}^{1 s}=\frac{\left(V_{N}-V_{r}\right)\left(V_{N}-V_{r}^{*}\right)\left(V_{N}-1 / V_{r}\right)\left(V_{N}-1 / V_{r}^{*}\right)}{-\left(V_{r}-1 / V_{r}\right)\left(V_{r}-1 / V_{r}^{*}\right)}, \quad r=\omega, \phi, \\
& H_{l}(V)=\frac{\left(V_{N}-V_{l}\right)\left(V_{N}-V_{l}^{*}\right)\left(V_{N}+V_{l}\right)\left(V_{N}+V_{l}^{*}\right)}{\left(V-V_{l}\right)\left(V-V_{l}^{*}\right)\left(V+V_{l}\right)\left(V+V_{l}^{*}\right)}, \\
& C_{l}^{1 s}=\frac{\left(V_{N}-V_{l}\right)\left(V_{N}-V_{l}^{*}\right)\left(V_{N}+V_{l}\right)\left(V_{N}+V_{l}^{*}\right)}{-\left(V_{l}-1 / V_{l}\right)\left(V_{l}-1 / V_{l}^{*}\right)}, \quad l=\omega^{\prime \prime}, \phi^{\prime \prime}, \omega^{\prime}, \phi^{\prime}, \\
& L_{k}(W)=\frac{\left(W_{N}-W_{k}\right)\left(W_{N}-W_{k}^{*}\right)\left(W_{N}-1 / W_{k}\right)\left(W_{N}-1 / W_{k}^{*}\right)}{\left(W-W_{k}\right)\left(W-W_{k}^{*}\right)\left(W-1 / W_{k}\right)\left(W-1 / W_{k}^{*}\right)}, \\
& C_{k}^{1 v}=\frac{\left(W_{N}-W_{k}\right)\left(W_{N}-W_{k}^{*}\right)\left(W_{N}-1 / W_{k}\right)\left(W_{N}-1 / W_{k}^{*}\right)}{-\left(W_{k}-1 / W_{k}\right)\left(W_{k}-1 / W_{k}^{*}\right)}, \quad k=\rho^{\prime \prime}, \rho^{\prime}, \rho, \\
& L_{r}(U)=\frac{\left(U_{N}-U_{r}\right)\left(U_{N}-U_{r}^{*}\right)\left(U_{N}-1 / U_{r}\right)\left(U_{N}-1 / U_{r}^{*}\right)}{\left(U-U_{r}\right)\left(U-U_{r}^{*}\right)\left(U-1 / U_{r}\right)\left(U-1 / U_{r}^{*}\right)}, \\
& C_{r}^{2 s}=\frac{\left(U_{N}-U_{r}\right)\left(U_{N}-U_{r}^{*}\right)\left(U_{N}-1 / U_{r}\right)\left(U_{N}-1 / U_{r}^{*}\right)}{-\left(U_{r}-1 / U_{r}\right)\left(U_{r}-1 / U_{r}^{*}\right)}, \quad r=\omega, \phi, \\
& H_{l}(U)=\frac{\left(U_{N}-U_{l}\right)\left(U_{N}-U_{l}^{*}\right)\left(U_{N}+U_{l}\right)\left(U_{N}+U_{l}^{*}\right)}{\left(U-U_{l}\right)\left(U-U_{l}^{*}\right)\left(U+U_{l}\right)\left(U+U_{l}^{*}\right)}, \\
& C_{l}^{2 s}=\frac{\left(U_{N}-U_{l}\right)\left(U_{N}-U_{l}^{*}\right)\left(U_{N}+U_{l}\right)\left(U_{N}+U_{l}^{*}\right)}{-\left(U_{l}-1 / U_{l}\right)\left(U_{l}-1 / U_{l}^{*}\right)}, \quad l=\omega^{\prime \prime}, \phi^{\prime \prime}, \omega^{\prime}, \phi^{\prime}, \\
& L_{k}(X)=\frac{\left(X_{N}-X_{k}\right)\left(X_{N}-X_{k}^{*}\right)\left(X_{N}-1 / X_{k}\right)\left(X_{N}-1 / X_{k}^{*}\right)}{\left(X-X_{k}\right)\left(X-X_{k}^{*}\right)\left(X-1 / X_{k}\right)\left(X-1 / X_{k}^{*}\right)} \\
& C_{k}^{2 v}=\frac{\left(X_{N}-X_{k}\right)\left(X_{N}-X_{k}^{*}\right)\left(X_{N}-1 / X_{k}\right)\left(X_{N}-1 / X_{k}^{*}\right)}{-\left(X_{k}-1 / X_{k}\right)\left(X_{k}-1 / X_{k}^{*}\right)}, \quad k=\rho^{\prime}, \rho, \\
& H_{\rho^{\prime \prime}}(X)=\frac{\left(X_{N}-X_{\rho^{\prime \prime}}\right)\left(X_{N}-X_{\rho^{\prime \prime}}^{*}\right)\left(X_{N}+X_{\rho^{\prime \prime}}\right)\left(X_{N}+X_{\rho^{\prime \prime}}^{*}\right)}{\left(X-X_{\rho^{\prime \prime}}\right)\left(X-X_{\rho^{\prime \prime}}^{*}\right)\left(X+X_{\rho^{\prime \prime}}\right)\left(X+X_{\rho^{\prime \prime}}^{*}\right)}, \\
& C_{\rho^{\prime \prime}}^{2 v}=\frac{\left(X_{N}-X_{\rho^{\prime \prime}}\right)\left(X_{N}-X_{\rho^{\prime \prime}}^{*}\right)\left(X_{N}+X_{\rho^{\prime \prime}}\right)\left(X_{N}+X_{\rho^{\prime \prime}}^{*}\right)}{-\left(X_{\rho^{\prime \prime}}-1 / X_{\rho^{\prime \prime}}\right)\left(X_{\rho^{\prime \prime}}-1 / X_{\rho^{\prime \prime}}^{*}\right)},
\end{aligned}
$$


mean that in the first case the resonance location is found in front of the effective inelastic square root branch point and in the second case the resonance location is found behind the effective inelastic square root branch point.

This model corresponds to nowadays experimentally confirmed nine neutral vector-mesons [2] $\rho(770), \omega(782)$, $\phi(1020), \rho^{\prime}(1450), \omega^{\prime}(1420), \phi^{\prime}(1680), \rho^{\prime \prime}(1700), \omega^{\prime \prime}(1650)$, $\phi^{\prime \prime}(2170)$; with quantum numbers of the photon and is in fact a well-matched unification of pole contributions of unstable vector mesons with cut structure in the complex plane $t$, whereby these cuts represent so-called continua contributions generated by exchange of more than one particle in the corresponding Feynman diagrams.

As a result in such model the shape of nucleon EM FFs is directly related to both, an existence of complex conjugate pairs of unstable vector-meson poles on three unphysical sheets of the four-sheeted Riemann surface in $t$ variable and also the cut contributions to the latter. The cuts simultaneously are securing FFs to be complex beyond the lowest possible thresholds on the positive real axis, as it is required by the FFs unitarity conditions.

Here we would like to note that the nucleon Dirac $F_{1}^{N}(t)$ and Pauli $F_{2}^{N}(t)$ FFs, as it is seen from the previous, are very suitable for theoretical description of the nucleon EM structure. However, for an extraction of experimental information on the nucleon EM structure from measured cross sections and polarizations, the nucleon Sachs EM FFs $G_{E}^{N}(t), G_{M}^{N}(t)$ are more suitable, which appear e.g. in the total cross section of $e^{+} e^{-} \rightarrow N \bar{N}$ process

$$
\begin{aligned}
& \sigma_{\text {tot }}\left(e^{+} e^{-} \rightarrow N \bar{N}\right)= \\
& \quad \frac{4 \pi \alpha^{2} C \beta_{N}(t)}{3 t}\left[\left|G_{M}^{N}(t)\right|^{2}+\frac{2 m_{N}^{2}}{t}\left|G_{E}^{N}(s)\right|^{2}\right],
\end{aligned}
$$

to be obtained by an integration of the differential cross section in [3] through the spatial angle, with $\beta_{N}(t)=\sqrt{1-\frac{4 m_{N}^{2}}{t}}$ and $C$ to be the Coulomb enhancement factor, without interference term, unlike Dirac and Pauli FFs.

The relations between the nucleon Sachs EM FFs $G_{E}^{N}(t), G_{M}^{N}(t)$ and the isoscalar and isovector parts of the nucleon Dirac and Pauli FFs are, for proton

$$
\begin{aligned}
G_{E}^{p}(t) & =\left[F_{1 s}^{N}(t)+F_{1 v}^{N}(t)\right]+\frac{t}{4 m_{p}^{2}}\left[F_{2 s}^{N}(t)+F_{2 v}^{N}(t)\right], \\
G_{M}^{p}(t) & =\left[F_{1 s}^{N}(t)+F_{1 v}^{N}(t)\right]+\left[F_{2 s}^{N}(t)+F_{2 v}^{N}(t)\right],
\end{aligned}
$$

and for neutron

$$
\begin{aligned}
& G_{E}^{n}(t)=\left[F_{1 s}^{N}(t)-F_{1 v}^{N}(t)\right]+\frac{t}{4 m_{n}^{2}}\left[F_{2 s}^{N}(t)-F_{2 v}^{N}(t)\right], \\
& G_{M}^{n}(t)=\left[F_{1 s}^{N}(t)-F_{1 v}^{N}(t)\right]+\left[F_{2 s}^{N}(t)-F_{2 v}^{N}(t)\right],
\end{aligned}
$$

with normalizations

$$
G_{E}^{p}(0)=1 ; G_{M}^{p}(0)=\mu_{p} ; G_{E}^{n}(0)=0 ; G_{M}^{n}(0)=\mu_{n} ;
$$

and

$$
\begin{aligned}
& F_{1 s}^{N}(0)=F_{1 v}^{N}(0)=\frac{1}{2} ; \\
& F_{2 s}^{N}(0)=\frac{1}{2}\left(\mu_{p}+\mu_{n}-1\right) ; \\
& F_{2 v}^{N}(0)=\frac{1}{2}\left(\mu_{p}-\mu_{n}-1\right),
\end{aligned}
$$

where $\mu_{N} N=p, n$ are the magnetic moments of the proton and neutron, respectively.

\section{Experimental Information on Nucleon EM FFs and Its Analysis}

The experimental information on the nucleon electric $G_{E}^{p}(t), G_{E}^{n}(t)$ and magnetic $G_{M}^{p}(t), G_{M}^{n}(t)$ FFs consists of the following different sets of the selected data

1. the ratio $\mu_{p} G_{E}^{p}(t) / G_{M}^{p}(t)$ in space-like region from polarization experiments $[4,5,6,7,8]$

2. $G_{E}^{p}(t)$ in space-like region [9]

3. $\left|G_{E}^{p}(t)\right|$ in time-like region; from experiment with $\left|G_{E}^{p}(t)\right|=\left|G_{M}^{p}(t)\right|$ assumption and [22]

4. $G_{M}^{p}(t)$ in space-like region $[10,11,12,13,14,15,16]$

5. $\left|G_{M}^{p}(t)\right|$ in time-like region $[17,18,19,20,21,22,23$, $24,25,26,27,45]$

6. $\left|G_{E}^{p}(t) / G_{M}^{p}(t)\right|$ in time-like region $[17,18,45]$

7. $G_{E}^{n}(t)$ in space-like region $[29,30,31,32,33,34,35$, 36]

8. $\left|G_{E}^{n}(t)\right|$ in time-like region; from experiment in which $\left|G_{E}^{n}(t)\right|=\left|G_{M}^{n}(t)\right|$ is assumed

9. $G_{M}^{n}(t)$ in space-like region $[29,36,37,38,39,40,41$, 42]

10. $\left|G_{M}^{n}(t)\right|$ in time-like region [28]

11. the ratio $\mu_{n} G_{E}^{n}(t) / G_{M}^{n}(t)$ in space-like region from polarization experiments on light nuclei [43, 44].

In a selection of the used data in the analysis the following criteria have been applied.

The polarization experiments [4-8] based on the simultaneous measurement of the transverse $P_{t}$ and longitudinal $P_{l}$ components of the recoil proton's polarization in the electron scattering plane of the polarization transfer process $\overrightarrow{e^{-}} p \rightarrow e^{-\vec{p}}$ provide very precise ratio $\mu_{p} \frac{G_{E}^{p}(t)}{G_{M}^{p}(t)}$ values. They clearly revealed the previous extraction of $G_{E}^{p}(t)$ by the Rosenbluth method to be not very promising. This is explained by the fact that with increased values of $(-t)$ the $G_{M}^{p}(t)$ in the differential cross section of the unpolarized process is starting to be considerably dominant [10-16]. Therefore in the analysis we have taken into account the ratio $\mu_{p} \frac{G_{E}^{p}(t)}{G_{M}^{p}(t)}$ data in space-like region from polarization experiments, the $G_{M}^{p}(t)$ values from unpolarized experiments and the experimental data on the separate $G_{E}^{p}(t)$ for higher values of $(-t)$ have been ignored.

Both separate proton EM FFs data in space-like region were used in the analysis only from MAINZ measurements [9] at very low values of $(-t)$ where the application of the 

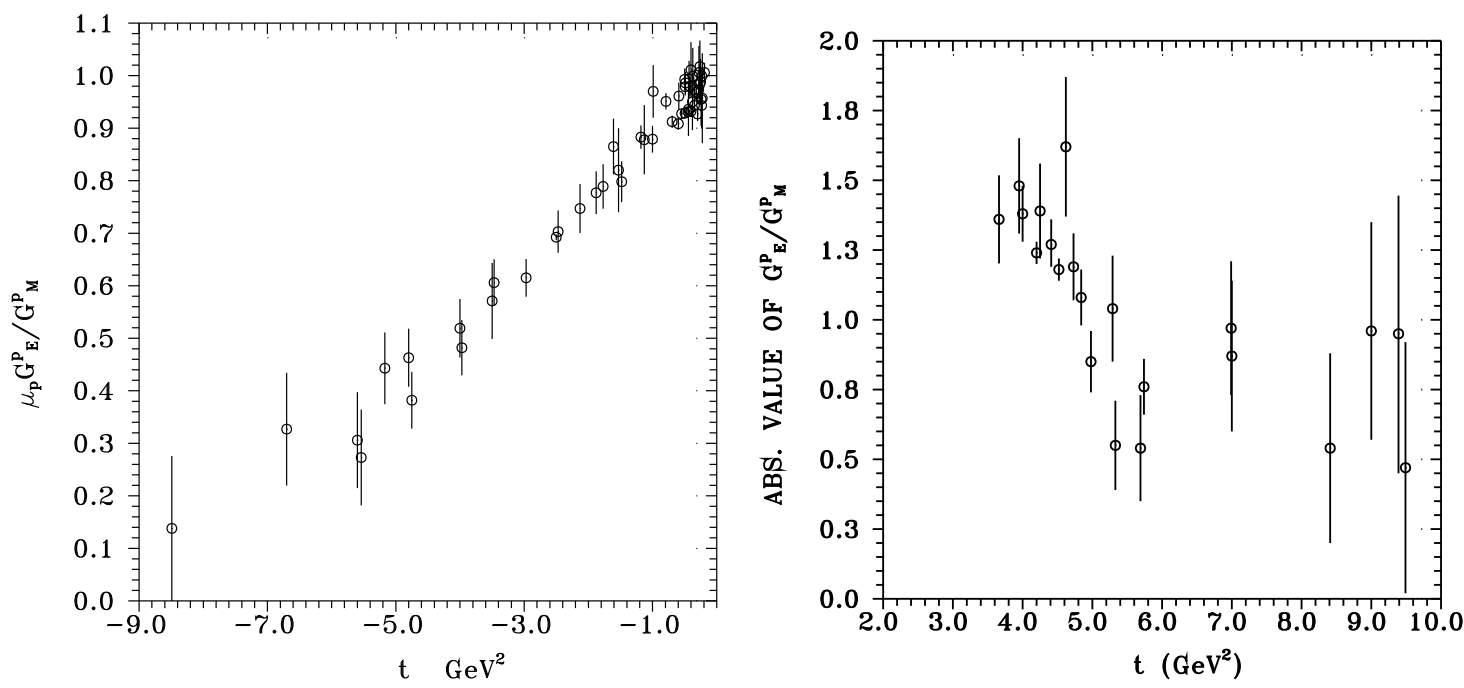

Figure 1. Experimental data on the ratios of the proton electric to magnetic FFs in space-like and time-like regions.
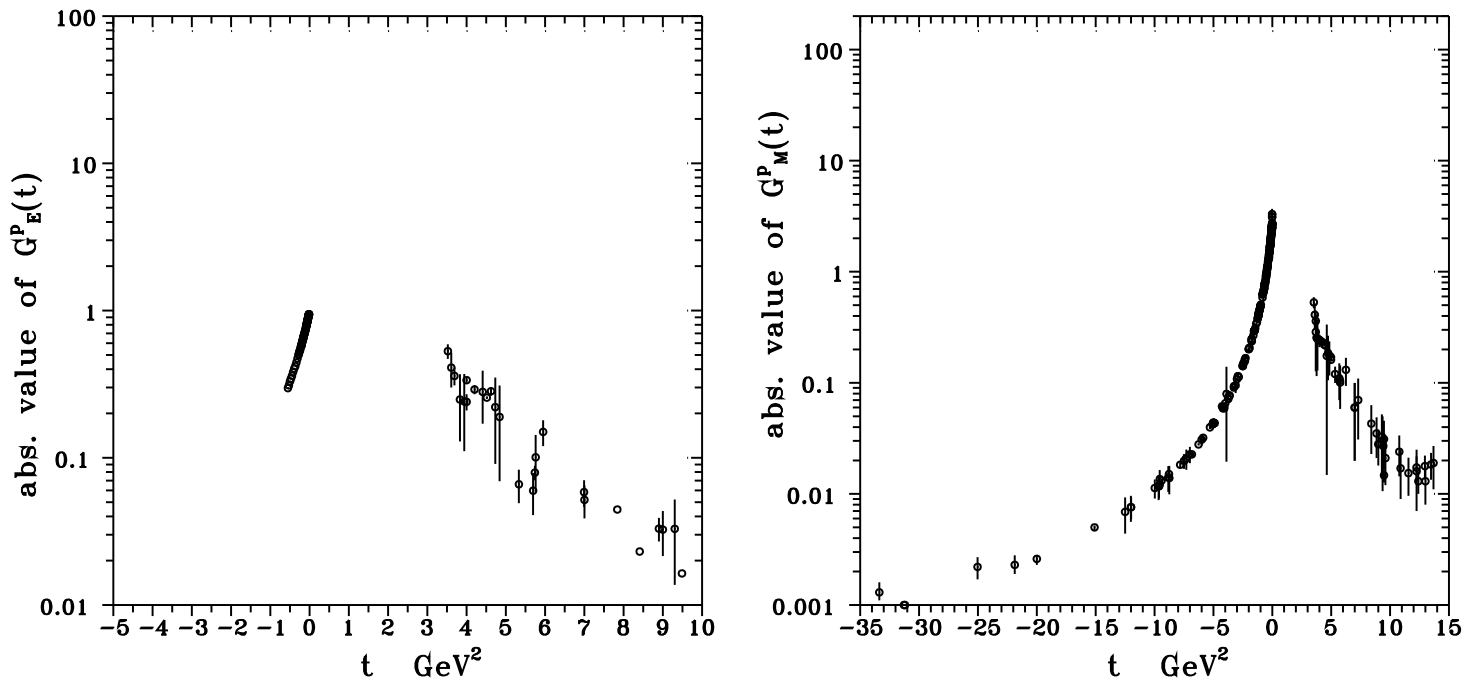

Figure 2. Experimental data on the proton electric and magnetic FFs in space-like and time-like regions.

Rosenbluth method has been justified.

In time-like region exact equality of both proton EM FFs is by definition just at the threshold of a creation of the protonantiproton pairs. If total cross section of the electron-positron annihilation into proton-antiproton pair has been measured not very far a way from this threshold, one could still draw out both FFs to be approximately equal and such data were utilized in the analysis.

If this total cross section is measured at enough high energies the electric proton FF is starting to give negligible contribution and practically only the values of $G_{M}^{p}(t)$ have been determined with errors to be used in our analysis.

The most reliable simultaneous determination of both proton EM FFs in time-like region has been achieved recently by the measurements of the proton angular distributions in the paper [45] at 16 different energies, which we have taken into account in the analysis too.

Poor neutron EM FFs data we have not classified as they are moreover charged by large errors.

All these data are graphically presented in Figures 1-4.

Results of a simultaneous analysis of these more or less 582 reliable experimental points on $G_{E}^{N}(t), G_{M}^{N}(t) N=p, n$ and their ratios by the nucleon electromagnetic structure $U \& A$ model to be given by the relations (3)-(6) through (15) and (16) with 12 free parameters are given in Table 1.

The corresponding behaviors of the proton and neutron electric and magnetic FFs on the base of these results and their comparison with experimental data are presented in Figures 5 and 6 by the dashed lines. 
Anna Zuzana Dubnickova and Stanislav Dubnicka: Proton Electromagnetic Form Factors Data Are in Disagreement with New Electron-Positron Annihilation into Proton-Antiproton Pair Total Cross Section Measurements
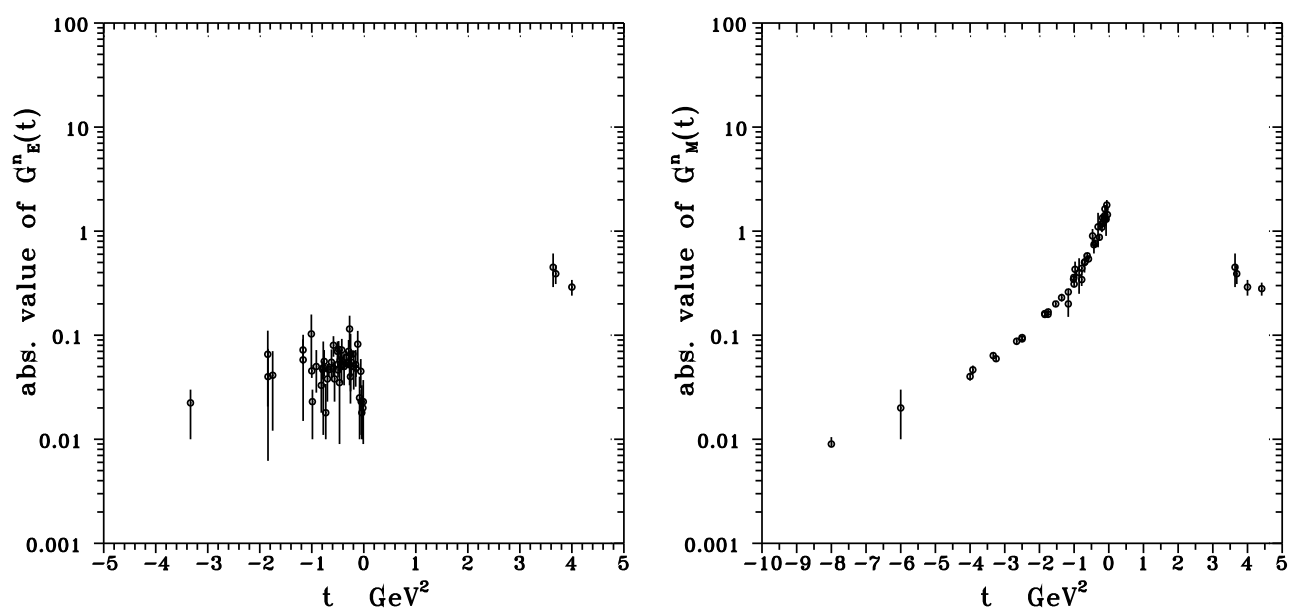

Figure 3. Experimental data on the neutron electric and magnetic FFs in space-like and time-like regions.

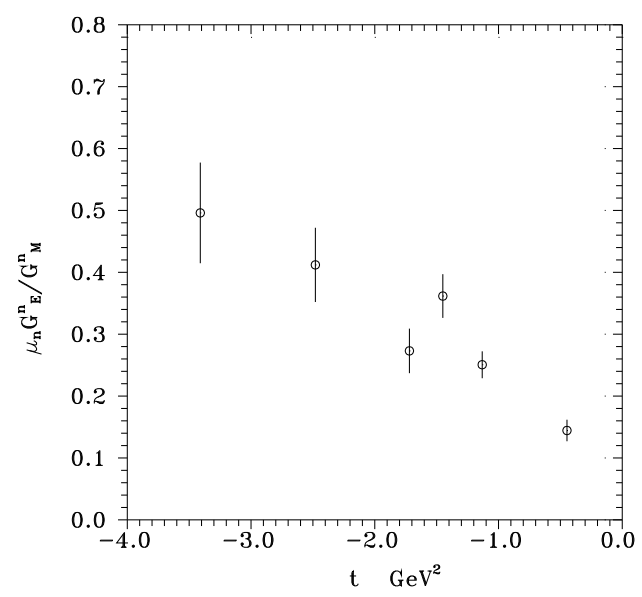

Figure 4. Data on the ratio $\mu_{n} G_{E}^{n}(t) / G_{M}^{n}(t)$ in the space-like region from polarization experiments on the light nuclei.

Table 1. Results of the analysis of the proton and neutron EM FFs data with $\chi^{2} / n d f=1.76$.

\begin{tabular}{ll}
\hline$t_{i n}^{1 s}=(1.4653 \pm 0.0542) \mathrm{GeV}^{2}$ & $t_{i n}^{1 v}=(2.9631 \pm 0.0072) \mathrm{GeV}^{2}$ \\
$t_{i n}^{2 s}=(1.8513 \pm 0.0049) \mathrm{GeV}^{2}$ & $t_{i n}^{2 v}=(2.3927 \pm 0.0039) \mathrm{GeV}^{2}$ \\
$\left(f_{\omega^{\prime} N N}^{(1)} / f_{\omega^{\prime}}\right)=-0.2780 \pm 0.0056$ & $\left(f_{\phi^{\prime} N}^{(1)} / f_{\phi^{\prime}}\right)=-0.5214 \pm 0.0030$ \\
$\left(f_{\omega N N}^{(1)} / f_{\omega}\right)=0.5988 \pm 0.0014$ & $\left(f_{\phi N N}^{(1)} / f_{\phi}\right)=-0.0287 \pm 0.0009$ \\
$\left(f_{\phi^{\prime} N N}^{(2)} / f_{\phi^{\prime}}\right)=0.0422 \pm 0.0156$ & $\left(f_{\omega N N}^{(2)} / f_{\omega}\right)=-0.4872 \pm 0.0828$ \\
$\left(f_{\phi N N}^{(2)} / f_{\phi}\right)=0.1216 \pm 0.0032$ & $\left(f_{\rho N N}^{(1)} / f_{\rho}\right)=-0.0602 \pm 0.0026$ \\
\hline
\end{tabular}

Table 2. Results of the analysis of only proton EM FFs data with $\chi^{2} / n d f=1.74$.

\begin{tabular}{ll}
\hline$t_{i n}^{1 s}=(1.6750 \pm 0.0363) \mathrm{GeV}^{2}$ & $t_{i n}^{1 v}=(2.9683 \pm 0.0091) \mathrm{GeV}^{2}$ \\
$t_{i n}^{2 s}=(1.8590 \pm 0.0023) \mathrm{GeV}^{2}$ & $t_{i n}^{2 v}=(2.4425 \pm 0.0208) \mathrm{GeV}^{2}$ \\
$\left(f_{\omega^{\prime} N N}^{(1)} / f_{\omega^{\prime}}\right)=-0.2937 \pm 0.0015$ & $\left(f_{\phi^{\prime} N N}^{(1)} / f_{\phi^{\prime}}\right)=-0.5298 \pm 0.0027$ \\
$\left(f_{\omega N N}^{(1)} / f_{\omega}\right)=0.6384 \pm 0.0025$ & $\left(f_{\phi N N}^{(1)} / f_{\phi}\right)=-0.0271 \pm 0.0005$ \\
$\left(f_{\phi^{\prime} N N}^{(2)} / f_{\phi^{\prime}}\right)=0.3075 \pm 0.0156$ & $\left(f_{\omega N N}^{(2)} / f_{\omega}\right)=0.1676 \pm 0.0377$ \\
$\left(f_{\phi N N}^{(2)} / f_{\phi}\right)=0.1226 \pm 0.0035$ & $\left(f_{\rho N N}^{(1)} / f_{\rho}\right)=-0.0802 \pm 0.0014$ \\
\hline
\end{tabular}



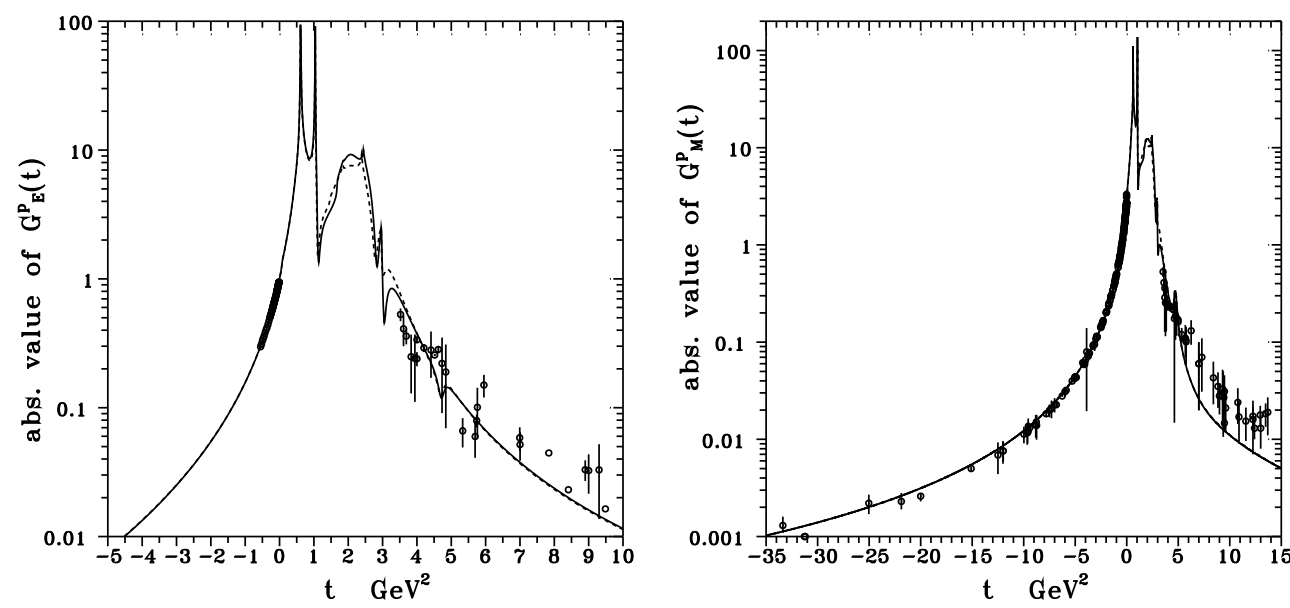

Figure 5. Prediction of proton electric and magnetic FFs behavior by the nucleon U\&A model [1] and its comparison with existing data.
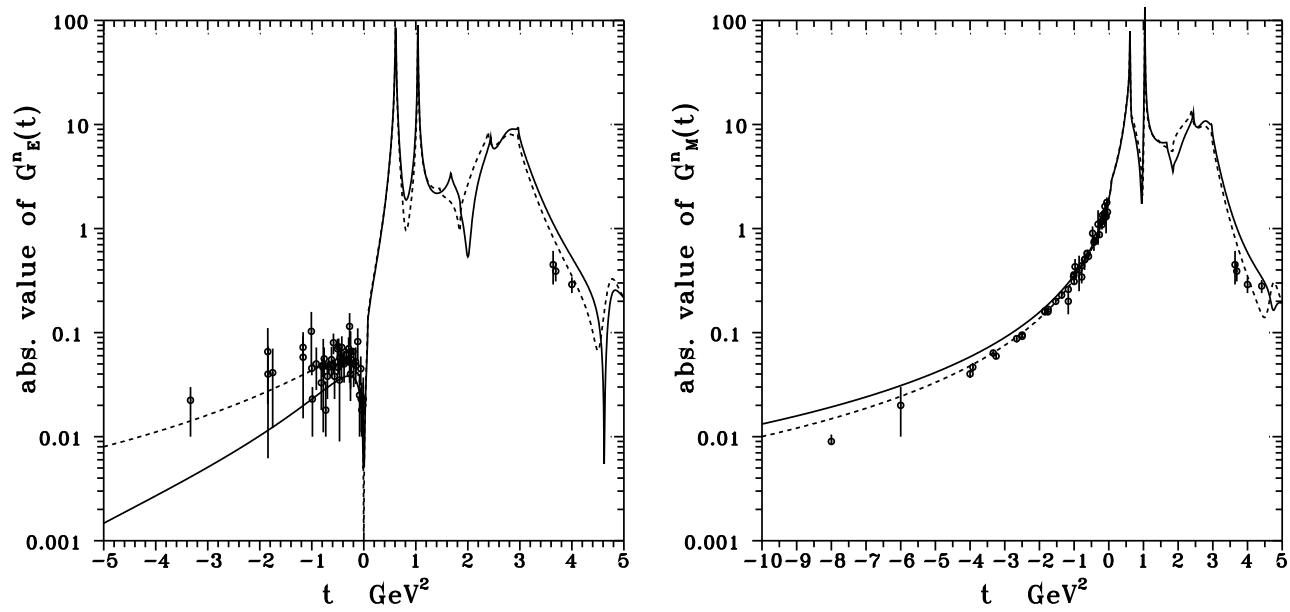

Figure 6. Prediction of neutron electric and magnetic FFs behavior by the nucleon U\&A model [1] and its comparison with existing data.

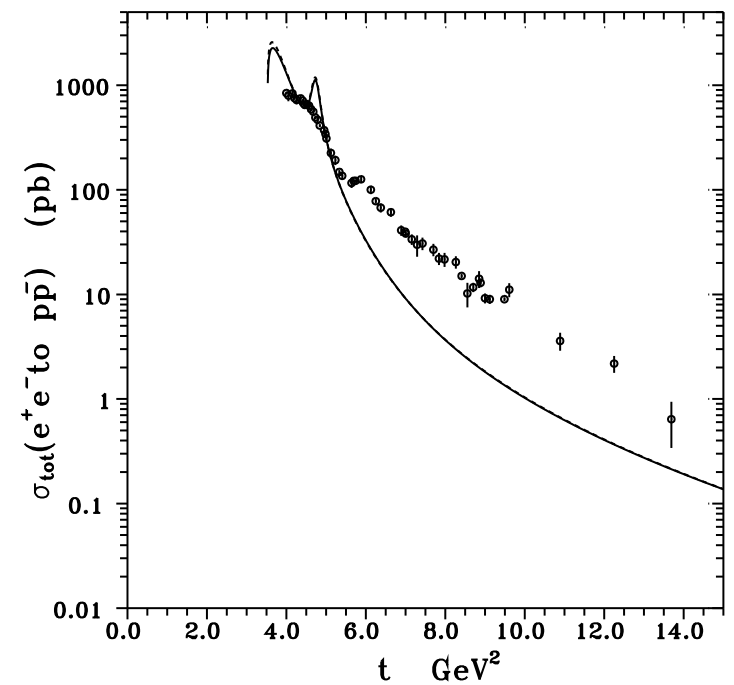

Figure 7. Prediction of $\sigma_{\text {tot }}\left(e^{+} e^{-} \rightarrow p \bar{p}\right)$ behavior by $G_{E}^{p}(t), G_{M}^{p}(t)$ from Figure 5 and its comparison with the recent data [45, 46] measured at the BEPCII collider: 
Substitution of these results on $G_{E}^{p}(t), G_{M}^{p}(t)$ given by dashed lines in Figure 5 into relation (14) to be modified for protons gives the theoretically predicted dashed curve in Figure 7 , where it is compared with very precise data $[45,46]$ on $\sigma_{t o t}\left(e^{+} e^{-} \rightarrow p \bar{p}\right)$ measured by BESIII Collaboration at the BEPCII collider exploiting the initial state radiation technique with an undetected photon.

The Figure 7 reveals a disagreement between the theoretically predicted $\sigma_{\text {tot }}\left(e^{+} e^{-} \rightarrow p \bar{p}\right)$ by using behaviors of $G_{E}^{p}(t), G_{M}^{p}(t)$ from Figure 5 and the experimental data on $\sigma_{\text {tot }}\left(e^{+} e^{-} \rightarrow p \bar{p}\right)[45,46]$ recently measured at the BEPCII collider.

There was our conjecture that the latter result could be caused by the neutron EM FFs data in the analysis as they are considerably less precise in comparison with the proton data.

In order to confirm or disprove our hypothesis we have excluded all neutron EM FFs data from the complete nucleon EM FFs compilation and the determination of the free parameters of the proton electromagnetic structure $U \& A$ model to be given by the relations (3)-(6) and (15) has been carried out by the analysis of only 459 reliable experimental data points with errors on the proton EM FFs.

The results from such analysis are presented in Table 2 .

The corresponding behaviors of the proton electric and magnetic FFs on the base of the parameters from Table 2 are presented in Figure 5 by full lines and their substitution into relation (14) to be modified for protons is given by full line in Figure 7. The covering of the previous dashed line by full line confirms our guess about the neutron EM FFs to be unreliable, which is confirmed also by full lines in Figure (6) for neutron EM FFs to be predicted by the parameters of the Table 2 .

The latter demonstrates a definite disagreement between the theoretical prediction of $\sigma_{\text {tot }}\left(e^{+} e^{-} \rightarrow p \bar{p}\right)$ by both $G_{E}^{p}(t), G_{M}^{p}(t)$ from Figure 5 represented by full and dashed lines and the experimental data on $\sigma_{t o t}\left(e^{+} e^{-} \rightarrow p \bar{p}\right)[45,46]$ recently measured at the BEPCII collider.

\section{Conclusions}

All existing data on the proton and neutron electromagnetic form factors, also on their ratios, have been collected and simultaneously described by means of the advanced nucleon EM structure $U \& A$ model [1], which is afterwards utilized for an investigation of the proton EM FFs data consistency with new precise data on $\sigma_{t o t}\left(e^{+} e^{-} \rightarrow p \bar{p}\right)[45,46]$.

A disagreement is revealed in Figure 7 between theoretical behavior of the $\sigma_{t o t}\left(e^{+} e^{-} \rightarrow p \bar{p}\right)$ calculated by means of the behaviors of the proton EM FFs and its recently measured data by BESIII Collaboration [45, 46], first by using behaviors of $G_{E}^{p}(t), G_{M}^{p}(t)$ obtained in the simultaneous analysis of data on proton and neutron EM FFs together, then this result is definitely confirmed also by using behaviors of $G_{E}^{p}(t), G_{M}^{p}(t)$ obtained in the analysis without the neutron EM FFs data.

How to explain the revealed inconsistency. There is no singularity of $G_{E}^{p}(t), G_{M}^{p}(t)$ FFs in the vicinity of $\mathrm{t}=5.5 \mathrm{GeV}^{2}$ point in the complex t-plane to be responsible for a fierce change of the $\sigma_{\text {tot }}\left(e^{+} e^{-} \rightarrow p \bar{p}\right)$ behavior (see Figure 7) in this energy. Therefore we are convinced that all existing experimental points of the total cross section $\sigma_{\text {tot }}\left(e^{+} e^{-} \rightarrow\right.$ $p \bar{p})$ in BESIII Collaboration measurements $[45,46]$ from $\mathrm{t}=5.5$ $\mathrm{GeV}^{2}$ up to $\mathrm{t}=13.49 \mathrm{GeV}^{2}$ are overestimated.

\section{Acknowledgements}

The support of the Slovak Grant Agency for Sciences VEGA, grant No. 2/0105/21, is acknowledged. The authors would like to thank Erik Bartoš and Cyril Adamuščín for valuable discussions.

\section{References}

[1] Adamuscin, C. Bartos, E. Dubnicka, S. Dubnickova, A.Z. (2016). Numerical Values of $f^{F}, f^{D}, f^{S}$ Coupling Constants in SU(3) Invariant Interaction Lagrangian of Vector-Meson Nonet with $1 / 2^{+}$Octet Baryons. Phys. Rev. C, 93, 055208.

[2] Tanabashi, M. et al. (2018). (Particle Data Group) Review of Particle Physics. Phys. Rev. D, 98, 03000.

[3] Baldini, R. Paceti, S. Zalo, A. Zichichi, A. (2009). Unexpected Features of $e^{+} e^{-} \rightarrow p \bar{p}$ and $e^{+} e^{-} \rightarrow \Lambda \bar{\Lambda}$ Cross Section Near Threshold. Eur. Phys. J. A, 39, 315.

[4] Jones, M. K. et al, (2000). $G_{E}^{p} / G_{M}^{p}$ Ratio by Polarization Transfer in Polarized e $\mathrm{p} \rightarrow$ e Polarized p. Phys. Rev. Lett. 84, 1398.

[5] Gayou, O. et al, (2002). Measurement of $G_{E}^{p} / G_{M}^{p}$ in Polarized-e $\mathrm{p} \rightarrow$ e Polarized-p to $\mathrm{Q}^{* * 2}=5.6-\mathrm{GeV}^{*} * 2$. Phys. Rev. Lett. 88, 092301.

[6] Punjabi, V. et al, (2005). Proton Elastic Form-Factor Ratios to $\mathrm{Q}^{* * 2}=3.5-\mathrm{GeV}^{* * 2}$ by Polarization Transfer. Phys. Rev. C, 71, 055202.

[7] Zhan, X. et al, (2011). High-Precision Measurement of the Proton Elastic Form Factor Ratio $\mu_{p} G_{E} / G_{M}$ at low $Q^{2}$. Phys. Lett. B, 705, 59.

[8] Pucket A.J.R. et al, (2011). Final Analysis of Proton Form Factor Ratio Data at $Q^{2}=4.04 .8$ and $5.6 \mathrm{GeV}^{2}$. Phys. Rev. C, 85, 045203.

[9] Bernauer, J. (2010). (MAMI), Measurement of the Elastic Electron-Proton Cross-Section and Separation of the Electric and Magnetic Form Factors in the $Q^{2}$ range from 0.004 to $1(\mathrm{GeV} / \mathrm{c})^{2}$. Ph.D. Thesis (Mainz: JohannesGutenberg-Univ).

[10] Janssens, T. et al, (1966). Proton Form Factors From Elastic Electron-Proton Scattering. Phys. Rev. 142, 922. 
[11] Berger, Ch. et al, (1971) Electromagnetic Form-Factors of the Proton at Squared Four Momentum Transfers Between 10-fm**-2 and $50 \mathrm{fm}^{* *}-2$. Phys. Lett. B, 35, 87.

[12] Bartel, W. et al, (1973). Measurement of Proton and Neutron Electromagnetic Form-Factors at Squared Four Momentum Transfers up to $3(\mathrm{GeV} / \mathrm{c})^{2}$. Nucl. Phys. B, $58,429$.

[13] Hoehler, G. et al, (1976). Analysis of Electromagnetic Nucleon Form-Factors. Nucl. Phys. B, 114, 505.

[14] Sill, A.F. et al, (1993). Measurements of elastic electron proton scattering at large momentum transfer. Phys. Rev. D, 48, 29.

[15] Walker, R.C. et al, (1994). Measurements of the Proton Elastic Form-Factors for $Q^{2}=1(\mathrm{GeV} / \mathrm{c})^{2}-3(\mathrm{GeV} / \mathrm{c})^{2}$ at SLAC. Phys. Rev. D, 49, 5671.

[16] Andivakis, L. et al, (1994). Measurements of the Electric and Magnetic Form-Factors of the Proton From $\mathrm{Q}^{* * 2}=$ $1.75-\mathrm{GeV} / \mathrm{c}^{* * 2} 2$ to $8.83-\mathrm{GeV} / \mathrm{c} * * 2$. Phys. Rev. D, 50, 5491.

[17] Ablikim, M. et al, (2015). Measurement of the Proton Form Factor by Studying $e^{+} e^{-} \rightarrow p \bar{p}$. (BESIII Collaboration), Phys. Rev. D, 91, 112004.

[18] Lees, J. et al, (2013). Study of $e^{+} e^{-} \rightarrow p \bar{p}$ Via InitialState Radiation at BABAR. (BaBar Collaboration), Phys. Rev. D, 87, 092005.

[19] Bassompierre, G. et al, (1983). Electron Positron Pair Production in Anti-P P Annihilation at Rest and Related Determination of the Electromagnetic Form-Factor of the Proton in the Timelike Region. Nuovo. Cim. A, 73, 347.

[20] Delcourt, B. et al, (1979). Study of the Reaction $e^{+} e^{-} \rightarrow$ $p \bar{p}$ in the Total Energy Range 1925-MeV - 2180-MeV. Phys. Lett. B, 86, 395.

[21] Bisello, D. et al, (1983). A Measurement of $e^{+} e^{-} \rightarrow p \bar{p}$ for $1975-M e V<\sqrt{s}<2250-M e V$. Nucl. Phys. B, $224,379$.

[22] Bardin, G. et al, (1994). Determination of the Electric and Magnetic Form-Factors of the Proton in the Timelike Region. (PS 170 Collaboration), Nucl. Phys. B, 411, 3.

[23] Armstrong, T.A. et al, (1993). Measurement of the Proton Electromagnetic Form-Factors in the Timelike Region at $9 \mathrm{GeV}^{2}$ to $13 \mathrm{GeV}^{2}$. (E760 Collaboration), Phys. Rev. Lett. 70, 1212.

[24] Ambrogiani, M. et al, (1999) Measurements of the Magnetic Form-Factor of the Proton in the Timelike Region at Large Momentum Transfer. (E735 Collaboration), Phys. Rev. D, 60, 032002.
[25] M. Ablikim et al, (2005). Measurement of the Cross Section for $e^{+} e^{-} \rightarrow p \bar{p}$ at Center-of-Mass Energies From 2.0-GeV to 3.07-GeV. (BES Collaboration), Phys. Lett. B, 630, 14.

[26] B. Aubert et al, (2006). A Study of $e^{+} e^{-} \rightarrow p \bar{p}$ Using Initial State Radiation With BABAR. (BaBar Collaboration),Phys. Rev. D, 73, 012005.

[27] J. P. Lees et al, (2013). Measurement of the $e^{+} e^{-} \rightarrow p \bar{p}$ Cross Section in the Energy Range From 3.0 to $6.5 \mathrm{GeV}$. (BaBar Collaboration), Phys.Rev. D, 88, 072009.

[28] Antonelli, A. et al, (1998). The First Measurement of the Neutron Electromagnetic Form-Factors in the Timelike Region. (FENICE Collaboration), Nucl. Phys. B, 517, 3.

[29] K. M. Hanson et al, (1973). Large Angle Quasielastic Electron-Deuteron Scattering. Phys. Rev. D, 8, 753.

[30] R. W. Berard et al, (1973). Elastic Electron Deuteron Scattering. Phys. Lett. 47, 355.

[31] S. Galster et al, (1971). Elastic Electron-Deuteron Scattering and the Electric Neutron Form Factor at FourMomentum Transfers $5 \mathrm{fm}^{-2}<q^{2}<14 \mathrm{fm}^{-2}$. Nucl. Phys. B, 32, 221.

[32] W. Bartel et al, (1972). Electromagnetic Form-Factors of the Neutron at Squared Four-Momentum Transfers of 1.0 and $1.5(\mathrm{GeV} / \mathrm{c})^{2}$. Phys. Lett. B, 39, 407.

[33] D. I. Glazier et al, (2005). Measurement of the Electric Form-Factor of the Neutron at $\mathrm{Q}^{* *} 2=0.3-(\mathrm{GeV} / \mathrm{c}) * * 2$ to $0.8-(\mathrm{GeV} / \mathrm{c})^{* * 2}$. Eur. Phys. J. A, 24, 101.

[34] G. Warren et al, (2004). Measurement of the Electric Form-Factor of the Neutron at $Q^{2}=0.5$ and 1.0 $\mathrm{GeV}^{2} / \mathrm{c}^{2}$. Phys. Rev. Lett. 92, 042301.

[35] J. Golak et al, (2001). Extraction of Electromagnetic Neutron Form Factors Through Inclusive and Exclusive Polarized Electron Scattering on a Polarized ${ }^{3} \mathrm{He}$ target. Phys. Rev. C, 63, 034006.

[36] S. Rock et al, (1982). Measurement of Elastic Electron - Neutron Cross-Sections Up to $\mathrm{Q}^{* * 2}=10-(\mathrm{GeV} / \mathrm{c})^{* * 2}$. Phys. Rev. Lett. 49, 1139.

[37] H. Anklin et al, (1998). Precise Measurements of the Neutron Magnetic Form-Factor. Phys. Lett. B, 428, 248.

[38] G. Kubon et al, (2002). Precise Neutron Magnetic FormFactors. Phys. Lett. B, 524, 26.

[39] P. Markowitz et al, (1993). Measurement of the Magnetic Form Factor of the Neutron. Phys. Rev. C, 48, R5.

[40] A. Lung et al, (1993). Measurements of the Electric and Magnetic Form-Factors of the Neutron From Q**2 $=1.75-\mathrm{GeV} / \mathrm{c}^{* * 2} 2$ to $4-\mathrm{GeV} / \mathrm{c}^{* * 2}$. Phys. Rev. Lett. 70, 718. 
[41] E. E. W. Bruins et al, (1995). Measurement of the Neutron Magnetic Form-Factor. Phys. Rev. Lett. 75, 21.

[42] B. Anderson et al, (2007). Extraction of the Neutron Magnetic Form Factor From Quasielastic $\overrightarrow{H e}^{3}\left(\vec{e}, e^{\prime}\right)$ at $Q^{2}=0,1-0,6(\mathrm{GeV} / \mathrm{c})^{2}$. Phys. Rev. C, 75, 034003.

[43] B. Plaster et al, (2006). Measurements of the Neutron Electric to Magnetic Form-Factor Ratio G(En) / G(Mn) via the H-2(polarized-e, e-prime,polarized-n)H1 Reaction to $\mathrm{Q}^{* * 2}=1.45-(\mathrm{GeV} / \mathrm{c})^{* * 2}$. Phys. Rev. C, 73,025205 .
[44] S. Riordan et al, (2010). Measurements of the Electric Form Factor of the Neutron up to $Q^{2}=3.4 \mathrm{GeV}^{2}$ Using the Reaction ${ }^{3} \mathrm{He} \rightarrow\left(\vec{e}, e^{\prime} n\right) p p$. Phys. Rev. Lett. 105, 262302.

[45] M.Ablikin et al., (2020). Measurement of Proton Electromagnetic Form Factors in $e^{+} e^{-} \rightarrow p \bar{p}$ in the Energy Region 2.00 - 3.08 GeV. (BESIII Collaboration), Phys. Rev. Lett. 124, 042001.

[46] M.Ablikin et al., (2019). Study of the Process $e^{+} e^{-} \rightarrow$ $p \bar{p}$ via Initial State Radiation at BESIII. Phys. Rev. D, 99, 092002. 